\title{
Pacific
}

Journal of

Mathematics

ON PROPER SURJECTIONS WITH LOCALLY TRIVIAL LERAY SHEAVES

ROBERT JAY DAVERMAN AND DAVID FRED SNYDER

Volume $170 \quad$ No. 2

October 1995 


\section{ON PROPER SURJECTIONS WITH LOCALLY TRIVIAL LERAY SHEAVES}

\section{R.J. DAVERMAN AND D.F. SNYDER}

Let $f: X \rightarrow Y$ be a closed proper surjection whose Leray sheaves are locally constant through a given dimension $k$. Spectral sequences are used to analyze the cohomological connectivity and manifold properties of $Y$. Generally, when $Y$ has dimension at most $k$, it is a cohomology $k$-manifold over a given principal ideal domain $R$ if and only if $H^{q}\left(X, X-f^{-1} y ; R\right)$ is isomorphic to $\operatorname{Hom}_{R}\left(H^{q-k}\left(f^{-1} y ; R\right), R\right)$ for every $y \in Y$ and $q \leq k$. As a result, if $X$ is an orientable $(n+k)$-manifold, each $f^{-1} y$ has the shape of a closed, connected, orientable $n$ manifold, and $Y$ is finite dimensional, then $Y$ is a generalized $k$-manifold. Euler characteristic relationships involving $X, Y$, and the typical fiber $f^{-1} y$ are derived in case the Leray sheaves of $f$ are locally constant in all dimensions and the range has cohomologically finite type.

The main thrust of this paper is to establish the following:

Theorem . Let $\mathcal{G}$ be an upper semicontinuous decomposition of an orientable $(n+k)$-manifold into subcompacta having the shape of closed, orientable $n$ manifolds such that the decomposition space $B$ is finite-dimensional and the Leray sheaf of the decomposition map is locally constant through dimension $k-1$. Then $B$ is a generalized $k$-manifold with boundary. If the Leray sheaf is also locally constant in dimension $k$, then $B$ is a generalized $k$-manifold.

Even if the Leray sheaf is constant in all dimensions, such a map need not be an approximate fibration, as Example 5.3 of [D1] shows.

The paper is organized into three sections. The first of these merely establishes the terminology and symbolism used in the sequel. It is known by a result of J. Dydak [Dy] that $B$ is $L C^{1}$; moreover, $B$ is an ANR if, and only if, $B$ is cohomologically locally connected. The second section concentrates on establishing cohomology manifold properties for the image of a closed, proper map provided the Leray sheaf is locally constant through certain dimensions. The third section applies the results of Section 2, the above 
theorem being one consequence. It also presents an Euler characteristic result (for closed proper maps with locally constant Leray sheaves in all dimensions) which is akin to the one for orientable fibrations. The most satisfying payoff occurs in studying a proper map $f: M \rightarrow B$ defined on an arbitrary closed, orientable $(n+2)$-manifold $M$ whose point preimages, up to cohomological equivalence, are all copies of a closed orientable $n$-manifold $N$, for then $\chi(M)=\chi(B) \cdot \chi(N)$. This indicates, for example, that neither integral cohomology 4-spheres nor closed orientable 4-manifolds of odd Euler characteristic can be upper semicontinuously decomposed into copies of a fixed surface.

\section{Definitions.}

All spaces are assumed to be separable metric spaces. Let $\mathbf{Q}, \mathbf{Z}$, and $\mathbf{Z}_{\mathbf{p}}$ denote the, rationals, integers, and integers modulo $p$, respectively. Let $\Lambda=\{2,3, \ldots\} \cup\{\infty\}$; as we use this often as an index set we regard $\Lambda$ as a totally ordered set, where $\infty$ represents the maximal element. We use $R$ to denote a principal ideal domain without zero divisors.

For the definition of a stack (presheaf) or sheaf on a space $Y$ and most of the following terminology, see [Sw] or [Br1]. A stack on $Y$ naturally induces a sheaf on $Y$; conversely, a sheaf naturally induces a stack (the stack of sections) over $Y$. The only stacks considered in this paper are the Leray stacks. Let $f: X \rightarrow Y$ be a continuous function; for any $q \geq 0$ and $U$ open in $Y$, define $S_{q}(U)=\check{H}^{q}(U ; R)$, the Leray stack in dimension $q$ of $f$ over $R$ on $Y$. We define $\mathcal{H}^{q}[f ; R]$ to be the sheaf induced by $S_{q}$ and $\mathcal{H}^{*}[f ; R]$ to be the "graded sheaf" $\left\{\mathcal{H}^{q}[f ; R] \mid q \geq 0\right\}$. When, by context, both $f$ and $R$ are understood, we may write $\mathcal{H}^{q}$ and $\mathcal{H}^{*}$ without ambiguity. Recall that a sheaf is trivial if it is equivalent, in the category of sheaves, to a constant sheaf $K \times Y$, where $K$ is an $R$-module; a sheaf $\mathcal{S}$ is locally trivial if for each $y \in Y$ there is a neighborhood $U$ of $y$ in $Y$ such that $\left.\mathcal{S}\right|_{U}$ is trivial.

Given an upper semicontinous decomposition (uscd) $\mathcal{G}$ of a space $M$, we let $B$ denote the decomposition space $M / \mathcal{G}$ and $\pi$ the decomposition map [D3]. For $A \subseteq B$, we follow the notation of James [J] and let $M_{A}=\pi^{-1}(A)$; if $b \in B$ then $M_{b}=\pi^{-1}(b)$.

We say that $\mathcal{G}$ (or $\pi$ ) is sheaf-trivial over $R$ in dimension $k$ (resp. locally sheaf-trivial over $R$ in dimension $k$ ), denoted $k-S T_{R}$ (resp. $k-L S T_{R}$ ), if $\mathcal{H}^{k}[\pi ; R]$ is trivial (resp. locally trivial) over the decomposition space. We say that $\mathcal{G}$ is sheaf-trivial (resp. locally sheaf-trivial) over $R$ through dimension $k$ denoted $S T_{R}^{k}$ (resp. $L S T_{R}^{k}$ ), if $\mathcal{G}$ is $l-S T_{R}$ (resp. $l-L S T_{R}$ ) for all $0 \leq l \leq k$. Furthermore, $\mathcal{G}$ is weakly locally sheaf-trivial over $R$, denoted $L S T_{R}^{\omega}$, if $\mathcal{G}$ is $S T_{R}^{k}$ for all $k \geq 0$. Finally, $\mathcal{G}$ is strongly locally sheaf-trivial over $R$, denoted 
$L S T_{R}^{\infty}$, if each point of the decomposition space has a neighborhood over which $\mathcal{H}^{q}[\pi ; R]$ is trivial, for all $q \geq 0$. If the subscript " $\mathrm{R}$ " is omitted, then $R=\mathbf{Z}$ is assumed.

There are three definitions of sheaf cohomology (categorical, Alexander and Cech), all of which agree for a given sheaf and the type of supports we consider; moreover, if the space is homologically locally connected (for example, an ANR) and the sheaf is trivial, then each of these theories agrees with the singular theory [Sw]. Thus, cohomology with coefficients in the Leray stack, or in the Leray sheaf, is unambiguously defined.

Since many of the spaces studied have potentially bizarre local structure, Cech cohomology is the theory employed throughout.

To prevent possible confusion, we spell out that the notation $\operatorname{Hom}_{R}(A, B)$ means the $R$-module of all $R$-homomorphisms $A \rightarrow B$.

A space $X$ is cohomologically locally connected in dimension $i$ with respect to $R$, denoted $i-c l c_{R}$, if for all $x \in X$ and all neighborhoods $U$ of $x$ in $X$, there is a neighborhood $V \subseteq U$ of $x$ such that $H^{i}(U ; R) \rightarrow H^{i}(V ; R)$ has finitely generated image. A space $X$ is cohomologically locally connected through dimension $i$ with respect to $R$, denoted $c l c_{R}^{i}$, if it is $j-c l c_{R}$ for all $0 \leq j \leq i$. A space $X$ is cohomologically locally connected with respect to $R$, denoted $c l c_{R}^{\omega}$, if it is $j-c l c_{R}$ for all $j \geq 0$. A space $X$ is strongly cohomologically locally connected with respect to $R$, denoted $c l c_{R}^{\infty}$, if for all $x \in X$ and all neighborhoods $U$ of $x$ in $X$, there is a neighborhood $V \subseteq U$ of $x$ such that $H^{i}(U ; R) \rightarrow H^{i}(V ; R)$ has finitely generated image for all $i \geq 0$. Note that a finite-dimensional metrizable space is $c l c_{R}^{\omega}$ if, and only if, it is $c l c_{R}^{\infty}$; in this case, we omit the superscript and say simply that the space is $\operatorname{clc}_{R}$. If the subscript " $R$ " is omitted, then $R=\mathbf{Z}$ is assumed.

A cohomology $k$-manifold over $R$ is a space $X$ of finite cohomological dimension with respect to $R$ [Na, p. 247] such that, for every $x \in X$, $H^{i}(X, X-x ; R)$ is trivial when $i \neq k$ and is isomorphic to $R$ when $i=k$. A cohomology $k$-manifold with boundary is a space $X$ such that for each $x \in X, H^{*}(X, X-x ; R)$ either is trivial or satisfies the requirements of a cohomology $k$-manifold. Following standard practice, we use $\partial X$ to denote $\left\{x \in X \mid H^{*}(X, X-x ; R)=0\right\}$. Older references, it should be mentioned, typically make it a requirement of the definition that $\partial X$ be closed relative to $X$, but W. J. R. Mitchell [Mi] has shown this always holds. A generalized $k$-manifold [-with-boundary] over $R$ is a cohomology $k$-manifold [-with-boundary] over $R$ which is, in addition, a $k$-dimensional ANR. For $k=0,1,2$, a generalized $k$-manifold is, in fact, locally Euclidean. However for $k \geq 3$, there are generalized $k$-manifolds that have no Euclidean patches; see [CD]. Even in the presence of such pathology, generalized manifolds are locally orientable [Br2]. 
The following Theorem's proof is found in [Br1, pp. 140-141].

Theorem (Leray-Grothendieck). For a closed map $f: X \rightarrow Y$, there is a first quadrant spectral sequence

$$
E_{2}^{p, q}=H_{\phi}^{p}\left(Y ; \mathcal{H}^{q}[f]\right) \Rightarrow H_{\phi(\psi)}^{p+q}(X) .
$$

\section{Remarks:}

1. For our purposes we need only consider the family $\psi$ of closed supports on $X$, and $\phi$ either the family of closed supports on $Y$ or $\phi=\{y\}$. In the former case $\phi(\psi)=\psi$, and in the latter $\phi(\psi)=\phi \mid \tilde{y}$, the closed subsets of $\tilde{y}=f^{-1} y$; in this latter case, if $f$ is also proper then $H_{\phi(\psi)}^{i}(X)=H^{i}(X, X-\tilde{y})$.

2. $E_{r+1}^{p, q}=\operatorname{ker}\left(d_{r}\right) / \operatorname{im}\left(d_{r}\right)$, where the differential $d_{r}^{p, q}: E_{r}^{p, q} \rightarrow E_{r}^{p+r, q-r+1}$ has bidegree $(r, 1-r)$.

3. $E_{r}^{p, q}=E_{r+1}^{p, q}=E_{r+2}^{p, q}=\cdots=E_{\infty}^{p, q}$ for $r \geq p+q+1$.

4. There is a filtration $0 \subset J_{0} \subset J_{1} \subset \cdots \subset J_{p}=H_{\phi(\psi)}^{p}(X)$, where $J_{0}=E_{\infty}^{p, 0}$ and $J_{i} / J_{i-1}=E_{\infty}^{p-i, i}, i \leq p$.

5. $[\mathbf{M c L}]$ has a quick introduction concerning calculation of spectral sequences.

6. $[\mathbf{B T}]$ is a wonderful beginner's guide to understanding the Leray sheaf, sheaf cohomology and the structure of this spectral sequence.

\section{Cohomological manifold properties of $B$.}

A standing hypothesis for this section is that $M$ is a connected separable metric space. Note that the elements of $\mathcal{G}$ in the following theorem are allowed to have infinite cohomological dimension.

Theorem 2.1. Let $\mathcal{G}$ be $L S T_{R}^{\omega}$, and suppose that $\mathcal{H}^{*}$ has finitely generated stalks in each dimension. Assume $M, M_{b}$ are connected and that $\operatorname{dim} B \leq k$. Then the following are equivalent:

1. $B$ is a cohomology $k$-manifold over $R$.

2. $H^{q}\left(M, M-M_{b}\right) \cong \operatorname{Hom}_{R}\left(H^{q-k}\left(M_{b}\right), R\right)$, for all $b \in B$ and $q \geq 0$.

Proof. Select $b \in B$. We let $\phi$ be the family $\{\emptyset,\{b\}\}$ of supports in the spectral sequence of $\pi$; as before, $\psi$ is the collection of closed subsets of $M$. We treat the case where $\mathcal{G}$ is $S T_{R}^{\infty}$, leaving the more general setting to the interested reader (hint: localize, use the excision property, and induct on $q$ ). (1) implies (2):

Since $B$ is a cohomology $k$-manifold, $H^{p}(B, B-b) \cong H_{\phi}^{p}(B)$ is trivial except for $p=k$, in which case it is $R$. Since $\mathcal{G}$ is $S T_{R}^{\infty}$, we can apply the 
Universal Coefficient theorem to $H_{\phi}^{p}\left(B ; \mathcal{H}^{q}\right)$ to see that $E_{2}^{p, q}$ is isomorphic to $\operatorname{Hom}_{R}\left(H^{q-k}\left(M_{b}\right), R\right)$ if $p=k$, and is trivial otherwise. So $E_{2} \cong E_{3} \cong \ldots$ and thus $E_{\infty}^{p, q}$ is trivial except when $p=k$, where $E_{\infty}^{k, q} \cong H_{\psi(\phi)}^{k+q}(M)$. Hence, $H^{q}\left(M, M-M_{b}\right) \cong H_{\psi(\phi)}^{q}(M) \cong E_{\infty}^{k, q-k} \cong E_{2}^{k, q-k} \cong H_{\phi}^{k}\left(B ; H^{q-k}\left(M_{b}\right)\right) \cong$ $\operatorname{Hom}_{R}\left(H^{q-k}\left(M_{b}\right), R\right)$ as promised.

(2) implies (1):

$E_{2}^{0, q}=H_{\phi}^{0}\left(B ; \mathcal{H}^{q}\left(M_{b}\right)\right) \cong 0$ since $B$ is connected.

A standard dimension argument [Na, pp. 244-247] gives $H^{p}(B, B-b) \cong 0$ for all $p>k$.

We now induct on $p$. For $p<k$, let $\Phi(p)$ denote the statement " $E_{r}^{p^{\prime}, q} \cong 0$ for all $q \geq 0, r \in \Lambda$ and $p^{\prime}+q \leq p$." Assume $\Phi(p-1)$, where $p<k$. Then $H^{p}(B, B-b) \cong H_{\phi}^{p}(B)=E_{2}^{p, 0} \cong E_{r}^{p, 0}$, the last isomorphism due to the image of $d_{r}^{p-r, r-1}$ being trivial for all $r$ by $\Phi(p-1)$; and so $E_{r}^{p, 0} \cong E_{\infty}^{p, 0}$. Thus $H^{p}(B, B-b) \cong H_{\psi(\phi)}^{p}(M)$, and, by hypothesis, the latter module is isomorphic to $\operatorname{Hom}_{R}\left(H^{p-k}\left(M_{b}\right), R\right)$, which is trivial since $p<k$ implies the coefficient group is trivial. Applying the Universal Coefficient Theorem [Sp, p. 246] to $E_{2}^{p^{\prime}, q} \cong H^{p^{\prime}}\left(B, B-b ; \mathcal{H}^{q}\right)$ for $0 \leq p^{\prime}+q \leq p$, we get $E_{2}^{p^{\prime} q} \cong 0$; thus $E_{r}^{p^{\prime}, q} \cong 0$ for all $0 \leq p^{\prime}+q \leq p$ and and $r \in \Lambda$. Hence, $\Phi(p)$ is true. In particular, by induction, $H^{p}(B, B-b) \cong 0$ for all $p<k$. Also note that we can now apply this argument for $p=k$ and conclude that $H^{k}(B, B-b)=H_{\psi(\phi)}^{k}\left(M, M-M_{b}\right) \cong \operatorname{Hom}_{R}\left(H^{0}\left(M_{b}\right), R\right) \cong R$. Thus, $B$ is a cohomology $k$-manifold over $R$.

The next result is a corollary to the proof of Theorem 2.1.

Corollary 2.2. Suppose $\mathcal{G}$ is $L S T_{R}^{k}$ and $\mathcal{H}^{l}$ has finitely generated stalks for each $l \leq k$. Assume $M, M_{b}$ are connected and $\operatorname{dim} B \leq k$. Then the following are equivalent:

1. $B$ is a cohomology $k$-manifold over $R$.

2. $H^{q}\left(M, M-M_{b}\right) \cong \operatorname{Hom}_{R}\left(H^{q-k}\left(M_{b}\right), R\right)$, for all $b \in B$ and $q \leq k$.

We can weaken the hypotheses of Corollary 2.2 somewhat, with a corresponding weakening of the conclusion.

Theorem 2.3. Let $\mathcal{G}$ be $L S T_{R}^{k-1}$, where $H^{l}$ has finitely generated stalks for $l \leq k-1$. Assume that $M, M_{b}$ are connected, $\operatorname{dim} B \leq k, H^{q}\left(M, M-M_{b}\right)=0$ for $q<k$, and $H^{k}\left(M, M-M_{b}\right) \subset R$. Then $B$ is a cohomology $k$-manifold with boundary over $R$.

Proof. Select $b \in B$. Exactly as in the proof of Theorem 2.1, we see that $H^{p}(B, B-b) \cong 0$ for $p<k$. Continuing in the same fashion, we find that

$$
H^{k}(B, B-b) \subset H^{k}\left(M, M-M_{b}\right) \subset R .
$$


According to a standing hypothesis, ideals in $R$ are either trivial or isomorphic to $R$, so $B$ is a cohomology $k$-manifold with boundary over $R$.

Example 2.4. The double $M$ of the mapping cylinder of the $2-1$ cover of $P^{2 n}$ by $S^{2 n}$ is a closed, orientable $(2 n+1)$-manifold which admits an obvious uscd $\mathcal{G}$ into two copies of projective $2 n$-space and an uncountable collection of $2 n$-spheres. Here $\mathcal{G}$ is $L C_{Z}^{1}$ for trivial reasons and $H^{1}(M, M-$ $\left.M_{b}\right) \cong H_{2 n}\left(M_{b}\right)$ is not always a copy of $Z$, being trivial for two values of b. As $B=M / \mathcal{G}$ clearly is a closed interval, this shows how boundary can occur in settings fulfilling less than the full strength of hypotheses in 2.2. Related examples involving arbitrary codimension $k<2 n$ arise from the map $\pi \times I d: M \times R^{k-1} \rightarrow[-1,1] \times R^{k-1}$, where the induced decomposition is $L S T_{R}^{k}$ for $R=\mathbf{Q}$ and $R=\mathbf{Z}_{p}$, provided $p$ is an odd prime; such examples reveal how boundary can arise for these values of $R$ even when $\mathcal{G}$ is $L S T_{R}^{k}$.

Corollary 2.5. Let $\mathcal{G}$ be $L S T_{R}^{k-1}$ where $H^{l}$ has finitely generated stalks for $l \leq k-1$. Assume that $M, M_{b}$ are connected, $\operatorname{dim} B \leq k, H^{q}\left(M, M-M_{b}\right)=0$ for $q<k$, and $H^{k}\left(M, M-M_{b}\right) \cong R$. Then $B$ is a cohomology $k$-manifold with boundary over $R$ and

$$
\partial B=\left\{b \in B \mid H_{b}^{0}\left(B ; \mathcal{H}^{k}\right)=R\right\}
$$

If we assume, in addition, that $\mathcal{H}^{k}$ is Hausdorff, then $\partial B$ is empty.

Proof. This requires just a minor addendum to the proof of Theorem 2.3. If $H^{k}(B, B-b) \cong 0$, then from the filtration of $H^{k}\left(M, M-M_{b}\right)$ induced by the spectral sequence, $R \cong H^{k}\left(M, M-M_{b}\right) \cong H_{b}^{0}\left(B ; \mathcal{H}^{k}\right)$. Obviously $\mathcal{H}^{k}$ Hausdorff implies $H_{b}^{0}\left(B ; \mathcal{H}^{k}\right)=0$, as then that sheaf has no sections whose support is a singleton.

Theorem 2.6. Suppose $N$ is a closed, $R$-orientable $n$-manifold; $M^{n+k}$ is an $R$-orientable $(n+k)$-manifold; $\mathcal{G}$ is a uscd of $M^{n+k}$ such that each $g \in \mathcal{G}$ has the shape of $N$ and $\mathcal{H}^{i}[\pi ; R]$ is locally constant for $0<i \leq k$; and $\operatorname{dim} B=k$. Then $\mathcal{H}^{n}[\pi ; R]$ is locally constant.

Proof. Just as in Theorem 2.1, $E_{2}^{p, q}=0$ unless $p=k$, so $E_{r}^{p, q}=0$ for $r \in \Lambda$, $p \neq k$. In the spectral sequence filtration of $H^{k}(B, B-b)$ we have

$$
J_{0}=E_{r}^{k, 0}=E_{2}^{k, 0} \cong H^{k}(B, B-b) \cong R \text { for all } r .
$$

Moreover, $J_{0} \cong J_{1} \cong \ldots \cong J_{k} \cong H^{k}\left(M, M-M_{b}\right) \cong H_{n}\left(M_{b}\right) \cong H^{n}\left(M_{b}\right)$, by duality. Local orientablility of $B$ implies local constancy of $\mathcal{H}^{n}[\pi ; R]$. 


\section{Discussion and Applications}

This section discusses the results of the previous sections in relation to the literature of manifold decompositions, and gives a few applications that are significant additions to the subject.

Lemma 3.1. Let $f: X \rightarrow Y$ be a closed, proper surjection whose pointinverses are path-connected FANRs, where $X$ is a locally compact ANR and $f$ is $L S T_{\mathbf{Z}}^{\omega}\left[\right.$ resp. $\left.L S T_{\mathbf{Z}}^{k-1}\right]$. Then $Y$ is $L C^{\omega}\left[\right.$ resp. $\left.L C^{k}\right]$. In particular, if $Y$ is finite dimensional [resp. $\operatorname{dim} Y \leq k]$, then $Y$ is an $A N R$.

Proof. A result of Dydak's [Dy] says that $Y$ is $L C^{\mathbf{1}}$. By Corollary 1 of [DyW2], $Y$ is $c l c_{\mathbf{Z}}^{\omega}$ [resp. $c l c_{\mathbf{Z}}^{k}$ ]. Hence, a fairly standard Hurewicz theorem (see [Sn, Lemma 1.4.8]) gives that $Y$ is $L C^{\omega}$ [resp. $L C^{k}$ ]. The concluding statement is implied by a well-known characterization of finite-dimensional ANRs [Hu].

Consider a usc decomposition $\mathcal{G}$ of an $(n+k)$-manifold $M$ into subcontinua having the shape of closed $n$-manifolds. When $k \leq 3$ the decomposition space $B=M / \mathcal{G}$ must be $k$-dimensional $[\mathbf{D 1}, \mathbf{D 2}, \mathbf{D W} 2]$; however, the discovery of dimension raising cell-like maps [Dr, DyW3] indicates that $\operatorname{dim} B$ can be infinite when $k>4$. From here on, therefore, we assume $B$ to be finitedimensional.

Next we present a cohomological variant of [DW2, Lemma 5.3]. Because that argument seems to require further elaboration, we give a complete proof here.

Theorem 3.2. Let $\mathcal{G}$ be a uscd of the orientable $(n+k)$-manifold $M$ into subcontinua having the shape of closed, $n$-manifolds, so that $B=M / \mathcal{G}$ is finite-dimensional and $\mathcal{G}$ is $L S T_{\mathbf{Z}}^{k-1}$.

Then $B$ is a generalized $k$-manifold (over $\mathbf{Z}$ ), possibly with boundary. Moreover, $\partial B=\emptyset$ if $\mathcal{H}^{k}[\pi ; \mathbf{Z}]$ is Hausdorff. In particular, if $\mathcal{G}$ is $L S T_{\mathbf{Z}}^{k}$, then $\partial B=\emptyset$, and $\mathcal{H}^{n}[\pi, \mathbf{Z}]$ is locally constant.

Proof. By Lemma 3.1, $B$ is an $A N R$.

Given $b \in B$, the Universal Coefficient Theorem yields

$H^{l}\left(M, M-M_{b}\right)=\operatorname{Hom}_{\mathbf{Z}}\left(H_{l}\left(M, M-M_{b}\right) ; \mathbf{Z}\right) \oplus \operatorname{Ext}\left(H_{l-1}\left(M, M-M_{b}\right) ; \mathbf{Z}\right)$.

Since $M$ is orientable, Alexander duality reveals that $H_{m}\left(M, M-M_{b}\right) \cong$ $H^{n+k-m}\left(M_{b}\right)$. So, for $l \leq k$, we can calculate that $H^{l}\left(M, M-M_{b}\right) \cong$ $H^{l-k}\left(M_{b}\right)$. Thus $B$ is a generalized manifold, possibly with boundary. As in the previous section, $\mathcal{H}^{k}[\pi ; \mathbf{Z}]$ Hausdorff implies $\partial B$ is empty. 
Finally, we note that $M$ need not be orientable as long as there is a orientable neighborhood of $M_{b}$, for every $b \in B$; also we may use any $P I D$ in place of $\mathbf{Z}$ if desired. The novelty of the result is for $k>2$, as Daverman and J.J. Walsh showed, without the sheaf-triviality restriction, that $B$ is a 2-manifold for $k=2$ [DW1]; and Daverman [D1] did the same for $k=1$.

The following result is useful for establishing the non-existence of certain types of manifold decompositions, and is similar to a result of [D5] in which $\pi$ is a $P L$ map, but not necessarily $L S T^{\omega}$.

Theorem 3.3 [Sp, p. 481]. Let $\mathcal{G}$ be an $L S T_{R}^{\omega}$ decomposition of a locally compact metric space $M$ into subcontinua having Euler characteristic definable over the field $R$ (or $R=\mathbf{Z}$ ) with $B=M / \mathcal{G}$ having definable Euler characteristic over $R$. Then the Euler characteristic over $R$ of $M$ can be defined and

$$
\chi(M)=\chi(B) \cdot \chi\left(M_{b}\right),
$$

where $M_{b}$ is the element of $\mathcal{G}$ over any $b \in B$.

The proof follows that of Spanier's fairly closely; he hypothesizes an orientable fibration rather than a decomposition of this type, but the algebra is the same. Moreover, we may apply the above result to get as a corollary:

Corollary 3.4. Let $\mathcal{G}$ be an $L S T_{R}^{\omega}$ decomposition of a closed, $R$-orientable (over $\mathbf{Z}$ or a field) $(n+k)$-manifold $M$ into subcontinua having the shape of $R$-orientable $n$-manifolds. Then, for any $b \in B$,

$$
\chi(M)=\chi(B) \cdot \chi\left(M_{b}\right) .
$$

Since $B$ is an $R$-cohomology $k$-manifold, this is immediate.

Lemma 3.5. Suppose $f: X \rightarrow R^{n}$ is a proper, surjective map, $H^{*}[f]$ is locally constant over $R^{n}$ - origin, and $f^{-1}$ (origin) is an ANR. Then $\chi\left(f^{-1}\left(R^{n}\right)\right)=\chi\left(f^{-1}(\right.$ origin $\left.)\right)$.

Proof. It suffices to show that the inclusion $f^{-1}$ (origin) $\rightarrow f^{-1}\left(R^{n}\right)$ induces a cohomology equivalence. The key step involves the following:

Claim. Let $V$ be the interior of the unit disk in $R^{n}$. Then the inclusion $f^{-1}(V-$ origin $) \rightarrow f^{-1}\left(R^{n}-\right.$ origin $)$ induces a cohomology isomorphism.

Just as in the proof of the claim in the midst of [DW2, Proposition 1.1], $H^{*}\left(V-\right.$ origin) and $H^{*}\left(R^{n}\right.$ - origin) are (naturally) isomorphic to $H^{*}\left(\left(R^{n}-\right.\right.$ origin) $\left.\times f^{-1}(z)\right), z \in R^{n}-$ origin. 
In light of the Claim, inspection of the cohomology ladder for the inclusion of pairs

$$
\left(f^{-1}(V), f^{-1}(V \text { - origin })\right)\left(f^{-1}\left(R^{n}\right), f^{-1}\left(R^{n}-\text { origin }\right)\right)
$$

quickly confirms that $H^{*}\left(f^{-1}\left(R^{n}\right)\right) \rightarrow H^{*}\left(f^{-1}(V)\right)$ is an isomorphism. By simply prearranging to have $f^{-1}(V)$ deformation retract to $f^{-1}$ (origin) in $f^{-1}\left(R^{n}\right)$, we obtain the required cohomology isomorphism and complete the proof of 3.5 .

Theorem 3.6. Suppose $N$ is a closed, orientable $n$-manifold and $\mathcal{G}$ is a uscd of a closed, orientable $(n+2)$-manifold $M$ into copies of $N$ (up to shape). Then $\chi(M)=\chi(B) \cdot \chi(N)$.

Proof. By [DW1] $B$ is a 2-manifold, and there exists a finite set $F=$ $b_{1}, \ldots, b_{m}$ such that $p: M-p^{-1}(F) \rightarrow B-F$ has locally constant Leray sheaf, in all dimensions. By Theorem 3.3

$$
\chi\left(M-p^{-1}(F)\right)=\chi(B-F) \cdot \chi(N)=(\chi(B)-k) \cdot \chi(N) .
$$

The various $b_{i} \in F$ have pairwise disjoint neighborhoods $U_{i}$, each homeomorphic to $R^{2}$. Lemma 3.5 assures $\chi\left(p^{-1}\left(U_{i}\right)=\chi(N)\right.$ for each $i$. Furthermore, $\chi\left(p^{-1}\left(U_{i}-b_{i}\right)\right)=\chi\left(U_{i}-b_{i}\right) \cdot \chi(N)=0$. Finally,

$$
\begin{aligned}
\chi(M) & =\chi\left(M-p^{-1}(F)\right)+\sum_{i=1}^{m} \chi\left(p^{-1}\left(U_{i}\right)\right)-\sum_{i=1}^{m} \chi\left(p^{-1}\left(U_{i}-b_{i}\right)\right) \\
& =(\chi(B)-m) \cdot \chi(N)+m \cdot \chi(N)-0 \\
& =\chi(B) \cdot \chi(N) .
\end{aligned}
$$

Corollary 3.7. If $G$ is uscd of a Z-cohomology $(2 n+2)$-sphere $M$ into continua having the shape of a fixed closed, orientable, $2 n$-manifold $F$, then $\chi(F)=1$.

Proof. Again, $B=M / G$ is a closed 2-manifold. Since $\pi$ induces an $H_{1}$ epimorphism, $B=S^{2}$. The conclusion follows directly from Theorem 3.6 and the fact that $\chi(M)=2$.

Corollary 3.8. There is no uscd of a Z-cohomology 4-sphere into continua having the shape of a fixed surface $F$.

Proof. If there were, the decomposition space would be a compact 2-manifold [D4]. Since an orientable $(n+k)$-manifold admits no uscd with ANR image into closed, non-orientable $n$-manifolds [DH, Theorems 2.10 and 3.1], $F$ must be orientable, and then Corollary 3.7 gives a contradiction. 
Corollary 3.9. If $M$ is a closed, orientable 4-manifold with $H^{1}(M ; \mathbf{Q})=0$, then there is no uscd of $M$ into continua having the shape of a surface $F$ with $\chi(F) \leq 0$; if, in addition, $\beta_{2}(M)>2$, then there is no uscd of $M$ into continua having the shape of any surface.

Proof. As in 3.8, $F$ would be orientable. Here again we would have $B=S^{2}$ or $B=P^{2}$, as $H^{1}(B ; \mathrm{Q})=0$. As then $\chi(M) \geq 2$ and $\chi(B) \geq 1$, this would yield $\chi(B) \cdot \chi(F) \leq 0<\chi(M)$ when $\chi(F) \leq 0$.

Corollary 3.10. There is no uscd of any closed, orientable 4-manifold of odd Euler characteristic into continua having the shape of a fixed surface $F$.

Proof. Here $\chi(F)$ must be even by the orientability argument of 3.8 .

Theorem 3.11. Suppose $N$ is a closed orientable $n$-manifold and $\mathcal{G}$ is a uscd of an orientable $(n+k)$-manifold $M$ such that each $g \in \mathcal{G}$ has the shape of an $A N R$ with $H^{*}(g ; \mathbf{Q}) \cong H^{*}(N ; \mathbf{Q}), \mathcal{H}^{n}$ is Hausdorff, and $\operatorname{dim} B<\infty$. Then $B$ is a Q-cohomology $k$-manifold.

Proof. Naturality of Poincaré duality implies that the Leray sheaf of $\pi$ is locally constant.

Corollary 3.12. Under the hypothesis of Theorem 3.11, assuming $M$ compact, $\chi(M)=\chi(N) \cdot \chi_{Q}(B)$.

Question: In Theorem 3.2, if we assume that the elements of $\mathcal{G}$ are orientable and $\mathcal{G}$ is $L S T_{\mathbf{Z}}^{k-1}$, is $\partial B$ necessarily empty? (Yes for $k=1,2$ [D1, DW1].)

\section{References}

[BT] R. Bott and L.W. Tu, An Introduction to Differential Forms in Algebraic Topology, Graduate texts in mathematics series 82, Springer-Verlag, New York 1982.

[Br1] G. Bredon, Sheaf Theory, McGraw-Hill, New York 1967.

[Br2] - Generalized manifolds revisited, in Topology of Manifolds, Proc. of Univ. of Ga. Topology of Manifolds Inst., 1969, J.C. Cantrell C.H. Edwards (eds.), Markham, Chicago 1970, pp. 461-469.

[CD] J.W. Cannon and R.J. Daverman, A totally wild flow, Indiana Univ. Math. J., 30 (1981), 371-387.

[D1] R.J. Daverman, Decompositions of manifolds into codimension one manifolds, Compositio Math., 55 (1985), 185-207.

[D2] The three dimensionality of certain codimension three decompositions, Proc. Amer. Math. Soc., 96 (1986), 175-179.

[D3] — Decompositions of Manifolds, Academic Press, Orlando 1986. 
[D4] Decompositions into codimension 2 submanifolds: the nonorientable case, Topology Appl., 24 (1986), 71-81.

[D5] — PL maps with manifold fibers, J. London Math. Soc., 45 (1992), pp. 180-192.

[DH] R.J. Daverman and L.S. Husch, Decompositions and approximate fibrations, Michigan Math. J., 31 (1984), 197-214.

[DW1] R.J. Daverman and J.J. Walsh, Decompositions of manifolds into codimension-2 submanifolds, Trans. Amer. Math. Soc., 288 (1985), 273-291.

[DW2] - Decompositions into submanifolds that yield generalized manifolds, Topology Appl., 26 (1987), 143-162.

[Dr] A.N. Dranishnikov, On a problem of P.S. Alexandrov, Math. USSR Sb., 63 (1989), 539-545.

[Dy] J. Dydak, On LC $C^{n}$-divisors, Topology Proc., 3 (1978), 319-333.

[DyS] J. Dydak and J. Segal, Local n-connectivity of decomposition spaces, Topology Appl., 18 (1984), 43-58.

[DyW1] J. Dydak and J.J. Walsh, Sheaves that are locally constant with applications to homology manifolds, in Geometric Topology and Shape Theory, S. Mardešić J. Segal (eds.), Lecture Notes in Mathematics 1283, Springer-Verlag, Berlin 1987, 65-87.

[DyW2] Cohomological local connectedness of decomposition spaces, Proc. Amer. Math. Soc., 107 (1989), 1095-1105.

[DyW3] - Infinite-dimensional compacta having cohomological dimension 2: an application of the Sullivan Conjecture, Topology, 32 (1993), 93-104.

[Hu] Sze-Tsen Hu, Theory of Retracts, Wayne State University Press, Detroit 1965.

[J] I.M. James, Fibrewise Topology, Cambridge tracts in mathematics 91, Cambridge University Press, Cambridge 1989.

[McL] John McCleary, User's Guide to Spectral Sequences, Publish or Perish, Wilmington, Delaware 1985.

[Mi] W.J.R. Mitchell, Defining the boundary of a homology manifold, Proc. Amer. Math. Soc., 110 (1990), 509-513.

[Na] Jun-Iti Nagata, Modern Dimension Theory, revised and extended edition, Heldermann Verlag, Berlin 1983.

[Sp] E.H. Spanier, Algebraic Topology, McGraw-Hill 1966.

[Sn] D.F. Snyder, Partially acyclic manifold decompositions yielding generalized manifolds, Trans. Amer. Math. Soc., 325 (1991), 531-571.

[Sw] R. Swan, Sheaf Theory, Univ. of Chicago Press, Chicago 1964.

Received February 1, 1993 and revised September 8, 1993.

UNIVERSITY OF TENNESSEE

KNOXVILLE, TN 37996-1300

E-MAIL ADDRESS: DAVERMAN@NOVELL.MATH.UTK.EDU

AND 



\section{CONTENTS}

N. Ben Amar, Tangential deformations on the dual of nilpotent special Lie

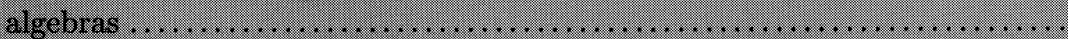

Martin Bendersky, Donald M. Davis and Mark Mahowald, $v_{1}$-periodic

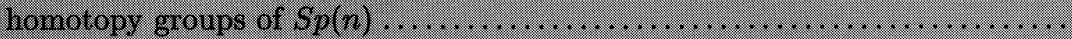

Georgia Benkart, Seok-Jin Kang, Kailash C. Misra, Indefinite Kac-

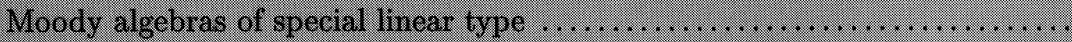

Robin Brooks and Charles Odenthal, Nielsen numbers for roots of maps

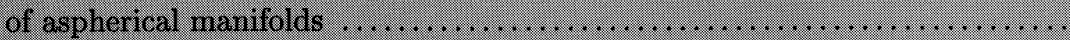

L.J. Bunce and J.D. Maitland Wright, Velocity maps in von Neumann

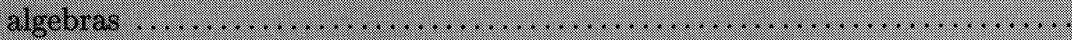

Bradley N. Currey, Smooth decomposition of finite multiplicity monomial representations for a class of completely solvable homogeneous spaces ...... R.J. Daverman and D.F. Snyder, On proper surjections with locally triv-

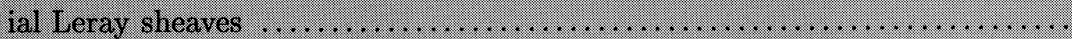

Patrick N. Dowling, Zhibao Hu and Mark A. Smith, MLUR renormings

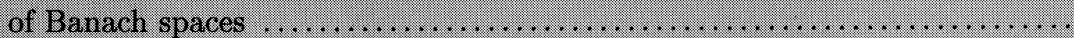
Tuval Foguel, Finite groups with a special 2-generator property ..........

Mourad E.H. Ismail and Mizan Rahman, Some basic bilateral sums and

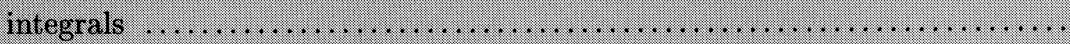

Wojciech Jaworski, Strong approximate transitivity, polynomial growth, and spread out random walks on locally compact groups $\ldots \ldots \ldots \ldots \ldots . . . . . .$.

N. Kutev and F. Tomi, Nonexistence and instability in the exterior Dirichlet problem for the minimal surface equation in the plane............

A. Nobile, Equisingularity Theory for Plane Curves With Embedded

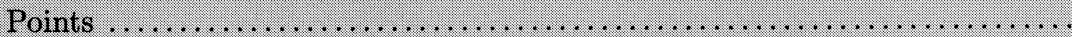

Dominikus Noll, Directional differentiability of the metric projection in Hilbert space 


\section{PACIFIC JOURNAL OF MATHEMATICS}

Volume $170 \quad$ No. $2 \quad$ October 1995

Tangential deformations on the dual of nilpotent special Lie algebras

297

NABIHA BEN AMAR

$v_{1}$-periodic homotopy groups of $S p(n)$

319

MaRTIN BENDERSKY, DONALD M. DAVIS and MARK MAHOWALD

Indefinite Kac-Moody algebras of special linear type

GeOrgia BenKart, SEOK-Jin KANG and KaILASh C. MisRa

Nielsen numbers for roots of maps of aspherical manifolds

405

ROBIN B. S. BROOKS and CHARLES ODENTHAL

Velocity maps in von Neumann algebras

L. J. BUNCE and JOHN DAVID MAITLAND WRIGHT

Smooth decomposition of finite multiplicity monomial representations for a class of 429 completely solvable homogeneous spaces

\section{BRADLEY CURREY}

On proper surjections with locally trivial Leray sheaves

ROBERT JAY DAVERMAN and DAVID FRED SNYDER

MLUR renormings of Banach spaces

PATRICK DOWLING, Zhibao Hu and MARK ANDREW SMIth

Finite groups with a special 2-generator property

TUVAL S. Foguel

Some basic bilateral sums and integrals

MOURAD ISMAIL and MIZAN RAHMAN

Strong approximate transitivity, polynomial growth, and spread out random walks on 517 locally compact groups

WOJCIECH JAWORSKI

Nonexistence and instability in the exterior Dirichlet problem for the minimal surface 535 equation in the plane

NiKolai KuteV and FriedRich TOMI

Equisingularity theory for plane curves with embedded points

Augusto Nobile

Directional differentiability of the metric projection in Hilbert space

DOMINIKUS NOLL 